\title{
Erratum to: Preliminary study on serum paraoxonase-1 status and chemokine (C-C motif) ligand 2 in hospitalized elderly patients with catheter-associated asymptomatic bacteriuria
}

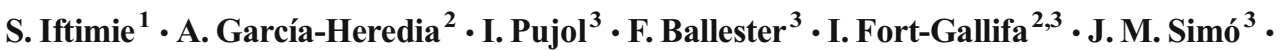 \\ J. Joven ${ }^{1} \cdot$ J. Camps ${ }^{1} \cdot$ A. Castro ${ }^{2}$
}

Published online: 19 July 2016

(C) Springer-Verlag Berlin Heidelberg 2016

Erratum to: Eur J Clin Microbiol Infect Dis

DOI: 10.1007/s10096-016-2679-8

The authors regret that Figure 1 of the original article was published online with error. The graph on the right was the same of that on the left. The corrected figure is presented below.
Also, the originally published article has been corrected.

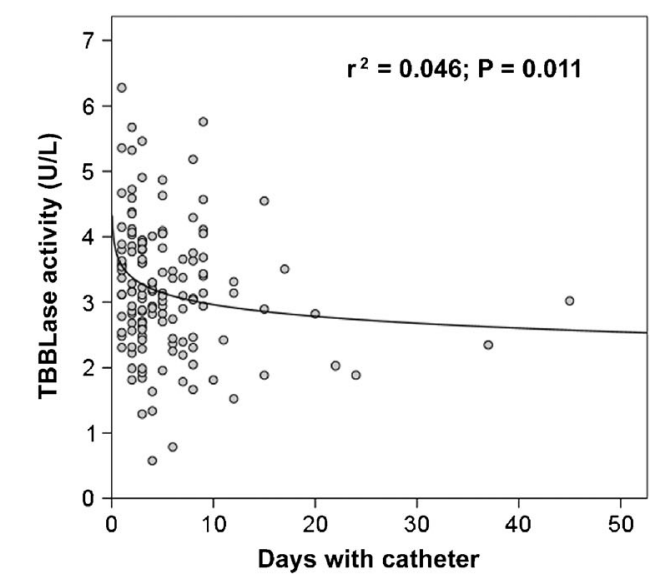

Fig. 1 Relationships between serum paraoxonase and TBBLase activities versus the number of days the urinary catheter was indwelling

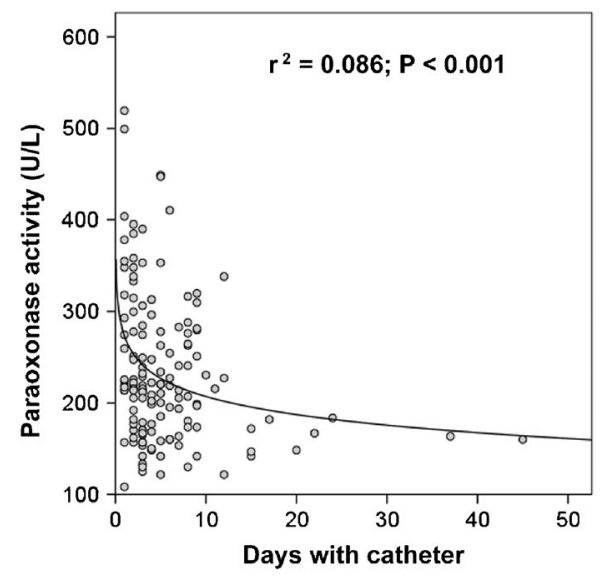

The online version of the original article can be found at http://dx.doi. org/10.1007/s10096-016-2679-8.

J. Camps

jcamps@grupsagessa.com

1 Department of InternalMedicine, Hospital Universitari de Sant Joan, Institut d'Investigació Sanitària Pere Virgili, Universitat Rovira i

Virgili, Unitat de Recerca Biomèdica, C. Sant Joan, s/n,

43201 Reus, Catalonia, Spain

2 Unitat de Recerca Biomèdica, Hospital Universitari de Sant Joan, Institut d'Investigació Sanitària Pere Virgili, Universitat Rovira i Virgili, C. Sant Joan, s/n, 43201 Reus, Catalonia, Spain

Laboratori de Referència Sud, Hospital Universitari de Sant Joan, Institut d'Investigació Sanitària Pere Virgili, Universitat Rovira i Virgili, Av. Comerç 42, 43204 Reus, Catalonia, Spain 